\title{
SELECTION OF INTRA PREDICTION MODES FOR INTRA FRAME CODING IN ADVANCED VIDEO CODING STANDARD
}

\author{
Manjanaik. $\mathbf{N}^{1}$, Manjunath. $\mathbf{R}^{2}$ \\ ${ }^{1}$ Electronics and Communication Engineering, Jain University, Bangalore, India. \\ ${ }^{2}$ Senior Domain Specialist, Philips Company, Bangalore, India. \\ manjubdt2009@gmail.com,manju_r_99@yahoo.com
}

\begin{abstract}
This paper proposes selection of Intra prediction modes for Intra frame coding in Advanced Video Coding Standard using Matlab. The proposed algorithm selects prediction modes for intra frame coding. There are nine prediction modes are there to predict the intra frame in AVC using Intra prediction, but all the prediction modes are not required for all the applications. Intra prediction is the first process of advanced video coding standard. It predicts a macro block by referring to its previous macro blocks to reduce spatial redundancy,appling all the prediction modes to predict intra frame it leads to more computational complexity is increased at the encoder of AVC. In the proposed algoriy $h m$, applied all the prediction modes $(0-8)$ for prediction of intra frame but only few modes such as mode0, mode1, mode2, mode4,mode6 gives good PSNR, high comprssion ratio and low bit rate. Out of these modes mode2 gives good PSNR, compression ratio and redced bit rate, mode5, mode7 and mode8 gives lower PSNR, low compression ratio and increased bitrate compared to mode0,mode1, mode2, mode4 and mode6. The simulation results are presented using Matlab. The PSNR, compressed ratio and bit rate achived for different quantization parameters of mother daughter frames, foreman frames was presented.
\end{abstract}

Keywords: AVC, PSNR, CAVLC, Macroblock, Prediction modes.

\section{INTRODUCTION}

Compression is the basic process of reduceing the size of data in order to save storge space and transmission band width.compression consist of removing redundancies (spatial,spectra and temporal) and encoding the true information in the form of appropriate to suite for applications. There are two compression techniques ie. Lossless and lossy techniques. In lossless the reconstured image after comprssion is identical to original image, this method achieve maximum compression ratio.To compress data, it is important to recognize redundancies in data in the form of coding redundancy, inter- pixel redundancy, and psycho-visual redundancy. Data redundancies occur when unnecessary data is used to represent source information. compression is achived when one or more of these types of redundancies are reduced.In lossy the reconstructed image is not identical to the original image ie there is loss in information.lossy method is capble of achiving a high comprssion compare to lossless method. Image compression/video coding is impotant in indusrial imaging, commercial and academic applications. Image /video coding plays a important role in multimedia. H.264/AVC is the latest video coding standard jointly developed by Joint video team which is organized by two international standards bodies ie the Internatonal Telecommunication Union-Telecommunications sector (ITUT) and International Organization for Standardization/
International Electro-technical Comission (ISO/IEC). This standard consists of various adavanced features(Intra prediction unit, integer transform, variable block mation estimation, entropy encoding, deblocking filter and coding tools.due to these features this standard achieves greater compression without sacrificing on video quality. Intra prediction unit is the first unit of H.264 standard. H.264 standard support intra prediction for diffent size ie $16 \times 16$ macro block as whole or $4 \times 4$ sub blocks.there are seventeen prediction modes for a macroblock, nine prediction modes for $164 \times 4$ luma sub macroblocks, four modes for a $16 \times 16$ luma macro block and four modes for two each two chorma $8 \times 8$ blocks. All possible combinations of intra prediction modes are used it lead to increase computational complexity of H.264 encoder and it is difficult to use for real time applications. All the modes are not relavant to all the applications, so that proposed algorithm is implemented for nine prediction mode.out of these mode few modes gives good PSNR, high compression ratio and low bit rate. These modes can use for suitable multi media applications.

\section{METHODOLOGY}

A raw recorded video in yuv format is used as an input file. The video is then decomposed into frames(I-frame). Each Intra frame is processed in terms of $4 \times 4$ sub macro block. For each Intra frame processing is done in order to get good video 
quality, high compression ratio and low bitrate as compared to previous video coding compression standards. Each Intra frame processing includes frame conversion i.e. RGB to YCBCR format down sampling i.e. (4:4:4 to 4:2:0), 4x4 sub macro block segmentation, all prediction modes (vertical, horizontal,DC, diagonal down left, horizontal down, diagonal down right, vertical left, horizontal up, vertical right etc) integer transformation, quantization, scaling and entropy encoding(cavlc). The video is reconstructed by inverse quantization, inverse transformation and deblocking filter.

\section{BLOCK DIAGRAM}

The block diagram of H.264 encoder for selection of Intra prediction modes is shown in fig. 1 . The h.264 encoder block consists of integer transform, quantization context adaptive variable length coding, inverse quantization, invese transformation, intra prediction unit and deblocking filter.

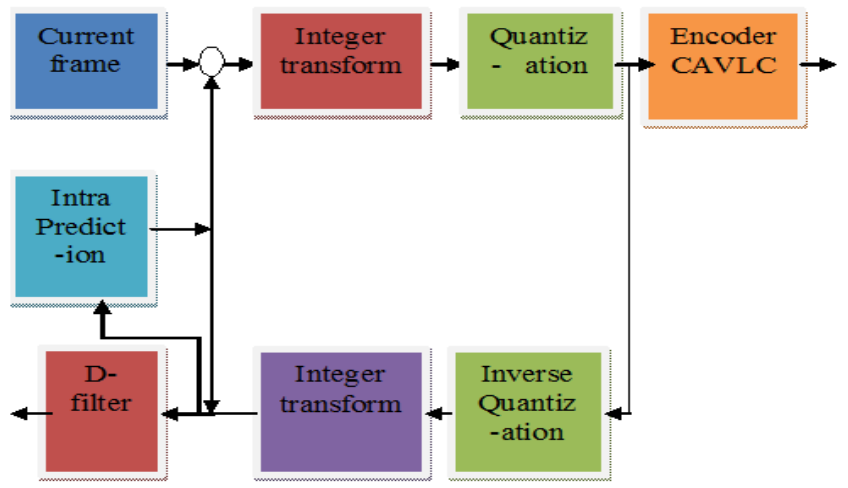

Fig.1 Block diagram of H.264 encoder

The block diagram of H.264/AVC encoder includes two dataflow paths, a forward path and a reconstruction path. An input frame is given for encoding. Every frame is processed in terms of a Macroblock (MB) of size 16x16 pixels. Each macroblock is further sub divided into $4 \times 4$ sub macroblock. Each $4 \times 4$ sub macroblock is encoded in intra prediction modes. A prediction macroblock $\mathrm{P}$ is formed based on a reconstructed block. In intra mode, $\mathrm{P}$ is formed from samples in the current block is based on previously reconstructed block. The prediction $\mathrm{P}$ is subtracted from the current macroblock to produce a residual or difference macroblock. This is transformed using integer transform and quantized using quantization block to give quantized transform coefficients. These coefficients are reordered and entropy encoded using context adaptive variable length coding (CAVLC) and the compressed bit stream is transmitted over a band-limited serial transmission channel. In the reconstruction path the quantized macroblock coefficients are decoded to reconstruct a frame for encoding of other macroblocks. The quantized coefficients are inverse quantized and inverse transformed to produce a difference macroblock. The prediction macroblock $\mathrm{P}$ is added to difference maroblock to create a reconstructed macroblock after a de-blocking filter, which improves the quality of the reconstructed frame[1-2].

\section{INTRA PREDICTION}

The H.264/AVC intra prediction unit achieves higher compression ratio and image quality compared with preivious standard(JPEG2000). The H.264 support different block sizes, it supports $4 \times 4$ and 16x16 block sizes for base line, main and extended profiles and $8 \times 8$ block size for high profile. There are nine prediction modes for $4 \times 4$ blocks, four for $16 \times 16$ blocks and and two for $8 \times 8$ blocks. All the prediction pixels are calculated based on the the reconstructed pixels of previously encoded neighbouring blocks. The prediction of $4 \times 4$ blocks is predicted based on the previously reconstructed pixels labelled (A-M) shown in Fig.2 the pixels (A-M) are reconstructed previously and consider as reference pixels for current block. The pixels labeled (a-m) are prediction pixels.

\begin{tabular}{|c|c|c|c|c|c|c|c|c|}
\hline $\mathrm{M}$ & A & B & $\mathrm{C}$ & $\mathrm{D}$ & $\mathrm{E}$ & $\mathrm{F}$ & $\mathrm{G}$ & $\mathrm{H}$ \\
\hline I & $\mathrm{a}$ & $\mathrm{b}$ & $\mathrm{c}$ & $\mathrm{d}$ & & & & \\
\hline $\mathbf{J}$ & $\mathrm{e}$ & $\mathrm{f}$ & $\mathrm{g}$ & $\mathrm{h}$ & & & & \\
\hline $\mathrm{K}$ & $\mathrm{i}$ & $\mathrm{j}$ & $\mathrm{k}$ & 1 & & & & \\
\hline $\mathrm{L}$ & $\mathrm{m}$ & $\mathrm{n}$ & 0 & $\mathrm{p}$ & & & & \\
\hline
\end{tabular}

Fig. 2. labeling of $4 \times 4$ prediction samples

Each $4 \times 4$ sub macroblock is predicted using eight directional prediction modes and one DC mode.The directional prediction modes are vertical, horizontal, diagonal down left, horizontal down, diagonal down right, vertical left, horizontal up, vertical right.For directional modes the predicted samples are formed from a weighted average of the perdiction samples A-M.For DC mode the predicted samples are formed by mean of samples A-D and I-L.The encoder select prediction mode for each $4 \times 4$ sub macroblock. The selection of best prediction mode is obtained by minimizing the residual encoded block and its prediction[3-5]. The fig.3 shows the intra prediction modes.

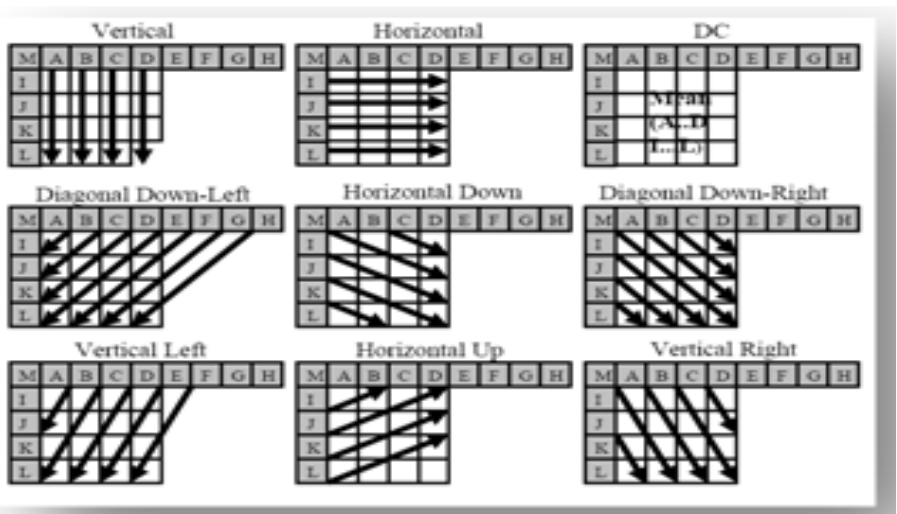

Fig.3. $4 \times 4$ intra prediction modes 
The equations of $4 \times 4$ sub macroblock for few prediction modes are:

Vertical

$\mathrm{a}=\mathrm{e}=\mathrm{i}=\mathrm{m}=\mathrm{A}$

$\mathrm{b}=\mathrm{f}=\mathrm{j}=\mathrm{n}=\mathrm{B}$

$\mathrm{c}=\mathrm{g}=\mathrm{k}=\mathrm{o}=\mathrm{C}$

$\mathrm{d}=\mathrm{h}=\mathrm{l}=\mathrm{p}=\mathrm{D}$

Horizontal

$\mathrm{a}=\mathrm{b}=\mathrm{c}=\mathrm{d}=\mathrm{I}$

$\mathrm{e}=\mathrm{f}=\mathrm{g}=\mathrm{h}=\mathrm{J}$

$\mathrm{i}=\mathrm{j}=\mathrm{k}=\mathrm{l}=\mathrm{K}$,

$\mathrm{m}=\mathrm{n}=\mathrm{o}=\mathrm{p}=\mathrm{L}$

DC

Mean of A-D and I-L,

for horizontal prediction sum of (A-D) and vertical prediction sum of (I-L).

Diagonal Down Left

$\mathrm{a}=(\mathrm{A}+2 \mathrm{~B}+\mathrm{C}+2)>>2$

$\mathrm{b}=\mathrm{e}=(\mathrm{B}+2 \mathrm{C}+\mathrm{D}+2)>>2$

$\mathrm{c}=\mathrm{f}=\mathrm{i}=(\mathrm{C}+2 \mathrm{D}+\mathrm{E}+2)>>2$

$\mathrm{d}=\mathrm{g}=\mathrm{j}=\mathrm{m}=(\mathrm{D}+2 \mathrm{E}+\mathrm{F}+2)>>2$

$\mathrm{h}=\mathrm{k}=\mathrm{n}=(\mathrm{E}+2 \mathrm{~F}+\mathrm{G}+2)>>2$

$\mathrm{i}=\mathrm{O}=(\mathrm{F}+2 \mathrm{G}+\mathrm{H}+2)>>2$

$\mathrm{p}=(\mathrm{G}+3 \mathrm{H}+2)>>2$.

\section{STEPS OF IMPLEMENTATION}

The following steps are to required to selection of intra prediction modes for intra frame coding in AVC.

- A recorded video in YUV CIF format as input file

- This file is decomposed into Intra frames (1-30)

- Each I-frame is divided into 16x16 macroblocks

- Eac h 16x16 macroblock further sub divided into 4x4 sub macroblocks

- A first $4 \times 4$ sub block is processed directly without using previously reconstructed block followed by integer transform, quantization, entropy encoding (cavlc) at encoder and reverse process at reconstruction path.

- Reconstruct a 4x4 subblock using inverse process (at reconstruction path)

- Obtain residual block by subtracting next 4x4 sub block with previously reconstructed sub block

- Residual of 4x4 sub block is integer transformed, quantized and entropy encoded at encoder and reverse process at reconstuction path.

- Finally measure PSNR, Compression ratio and Bit rate of intra frames with different QP for all prediction modes

\section{IMPLEMENTAION}

The proposed work is carried out using Matlab. The input is yuv sequences CIF format video file. For test purpose motherdaughter frame of resolution $352 \times 288$ and also other frames (foreman, news, etc) also taken, A Matlab program is written, which reads the yuv video file, extracts Intra frames. The next process involves reading a true RGB colour frame and convert into ycbcr format down sampling (4:4:4 to $4: 2: 0)$ to reduce bits of intra frame.ycber intra frame is divided into $4 \times 4$ sub block is processed directly by following usual procedure of forward path of H.264 encoder and reconstruct the processed block which serves as reference to the next sub block using basic reverse process in reconstruction path of h.264 encoder. Apply all the prediction modes to each sub block followed by, quantization, context adaptive variable length coder (CAVLC) to get compressed bit. At H.264 encoder in the reconstruction path, perfom reverse process to get reconstruct image and finally measure quality picture (PSNR).

\section{RESULTS AND DISCUSSION}

The proposed method, selection of intra prediction modes and best prediction mode for intra frame is done using Matlab. The test sequences are coded with only intra frames with frame rate 25 frames per second.The results obtained for the test yuv sequences in CIF format, five forman cif frames for quantization parameter 30 and ten mother-daughter cif frames for quantization parameter 35. Other quantization parametes[10, 25, 40, 45,51] also chosen (not shown in table).

Table-1

\begin{tabular}{|c|c|c|c|c|c|c|}
\hline \multicolumn{7}{|c|}{ PSNR, Compression ratio, Bit-rate, of foreman cif frames (1-5) with QP=30 } \\
\hline \multirow{2}{*}{ PSNR db } & Modes & Frame1 & Frame2 & Frame3 & Frame4 & Frame5 \\
\cline { 2 - 7 } & 0 & 31.75 & 31.7723 & 31.7593 & 31.7743 & 31.755 \\
\hline
\end{tabular}




\begin{tabular}{|c|c|c|c|c|c|c|}
\hline & 2 & 31.9123 & 31.9029 & 31.878 & 31.891 & 31.8734 \\
\hline & 3 & 31.3911 & 31.3794 & 31.3674 & 31.3834 & 31.3826 \\
\hline & 4 & 31.3611 & 31.3585 & 31.3511 & 31.359 & 31.3526 \\
\hline & 5 & 28.6738 & 28.6752 & 28.6804 & 28.6671 & 28.6521 \\
\hline & 6 & 30.5477 & 30.5331 & 30.5119 & 30.5188 & 30.5123 \\
\hline & 7 & 27.2161 & 27.2159 & 27.2144 & 27.2127 & 27.1954 \\
\hline & 8 & 26.6998 & 26.6965 & 26.6952 & 26.692 & 26.6837 \\
\hline \multirow{9}{*}{$\begin{array}{c}\text { Compression } \\
\text { Ratio (\%) }\end{array}$} & 0 & 75.5339 & 75.5365 & 75.5508 & 75.6185 & 75.4362 \\
\hline & 1 & 75.319 & 75.3607 & 75.319 & 75.2826 & 75.2435 \\
\hline & 2 & 76.8424 & 76.8776 & 76.7617 & 76.9245 & 76.7943 \\
\hline & 3 & 68.6198 & 68.7096 & 68.8906 & 68.7891 & 68.6549 \\
\hline & 4 & 66.7331 & 66.9635 & 66.9193 & 66.9245 & 66.9596 \\
\hline & 5 & 42.625 & 42.6003 & 42.6523 & 42.6185 & 42.6042 \\
\hline & 6 & 67.6641 & 67.6849 & 67.6589 & 67.8789 & 67.6875 \\
\hline & 7 & 59.1706 & 59.2604 & 59.2995 & 59.2396 & 59.1602 \\
\hline & 8 & 53.1146 & 53.1276 & 53.181 & 53.1758 & 53.1393 \\
\hline \multirow{9}{*}{ Bit rate } & 0 & 3.5839 & 3.5835 & 3.5814 & 3.5715 & 3.5982 \\
\hline & 1 & 3.6154 & 3.6093 & 3.6154 & 3.6207 & 3.6264 \\
\hline & 2 & 3.3922 & 3.3870 & 3.4040 & 3.3802 & 3.9928 \\
\hline & 3 & 4.5967 & 4.5835 & 4.5570 & 4.5719 & 4.5915 \\
\hline & 4 & 4.8730 & 4.8393 & 4.8458 & 4.8450 & 4.8399 \\
\hline & 5 & 8.4054 & 8.4081 & 8.4005 & 8.4054 & 8.4075 \\
\hline & 6 & 4.7367 & 4.7336 & 4.7374 & 4.7052 & 4.7332 \\
\hline & 7 & 5.9808 & 5.9677 & 5.9619 & 5.9707 & 5.9824 \\
\hline & 8 & 6.8679 & 6.8660 & 6.8582 & 6.8590 & 6.8643 \\
\hline
\end{tabular}

The table 1 shows comparison of nine intra prediction modes for formen cif frames $4 \times 4$ sub macroblocks with $\mathrm{QP}=30$ and table 2 shows that comparison of nine prediction modes for mother-daughter cif frames $4 \times 4$ sub macroblocks with $\mathrm{QP}=35$. The various figs (4-22) are simulation results (i.e. original frame, reconstructed frames and reconstructed frames with deblocking filter) of mother-daughter cif frames, foreman cif frames, news cif frames for nine intra prediction modes (0-8) and quantization parameters-30, 35. Quantization parameter 30 for foreman cif frames, news cif frames. Quantization parameter 30 mother-daughter cif frames. 
IJRET: International Journal of Research in Engineering and Technology eISSN: 2319-1163 | pISSN: 2321-7308

Table 2

\begin{tabular}{|c|c|c|c|c|c|c|c|c|c|c|c|}
\hline \multicolumn{12}{|c|}{ PSNR,Compression ratio, Bit-rate of mother-daughter_cif frames(1-10) with QP=35 } \\
\hline & $\begin{array}{c}\text { Mode } \\
s\end{array}$ & Frame1 & Frame2 & Frame3 & Frame4 & Frame5 & Frame6 & Frame7 & Frames & Frame9 & Frame10 \\
\hline \multirow{9}{*}{ PSNR in db } & 0 & 33.14123 & 33.1166 & 33.10555 & 33.13742 & 33.14686 & 33.13439 & 33.11761 & 33.1143 & 33.1006 & 33.10746 \\
\hline & 1 & 33.08717 & 33.05939 & 33.07195 & 33.099 & 33.09906 & 33.10453 & 33.09241 & 33.10064 & 33.07036 & 33.06456 \\
\hline & 2 & 33.20455 & 33.19078 & 33.19016 & 33.21944 & 33.22558 & 33.2023 & 33.18459 & 33.19254 & 33.17163 & 33.17553 \\
\hline & 3 & 32.67051 & 32.64043 & 32.66463 & 32.68794 & 32.68958 & 32.69664 & 32.65912 & 32.65468 & 32.66058 & 32.66091 \\
\hline & 4 & 32.69006 & 32.67825 & 32.6745 & 32.70011 & 32.70622 & 32.70534 & 32.67992 & 32.69209 & 32.66437 & 32.67972 \\
\hline & 5 & 30.34563 & 30.33966 & 30.34171 & 30.35221 & 30.36275 & 30.35223 & 30.33657 & 30.34927 & 30.34033 & 30.35441 \\
\hline & 6 & 32.69446 & 32.69688 & 32.69399 & 32.72496 & 32.74467 & 32.72919 & 32.70603 & 32.69553 & 32.70138 & 32.70141 \\
\hline & 7 & 28.53597 & 28.53009 & 28.54107 & 28.53057 & 28.53239 & 28.54504 & 28.54817 & 28.54717 & 28.54423 & 28.54054 \\
\hline & 8 & 27.78601 & 27.78477 & 27.789 & 27.7796 & 27.78141 & 27.7853 & 27.78161 & 27.78453 & 27.78259 & 27.79232 \\
\hline \multirow{9}{*}{$\begin{array}{c}\text { Compr ession } \\
\text { Ratio (\%) }\end{array}$} & 0 & 86.23307 & 86.14453 & 86.21875 & 86.23307 & 86.27865 & 86.21094 & 86.17188 & 86.11719 & 86.14063 & 86.13021 \\
\hline & 1 & 86.25651 & 86.22526 & 86.22656 & 86.21484 & 86.38021 & 86.3112 & 86.25 & 86.23177 & 86.23307 & 86.24089 \\
\hline & 2 & 86.71875 & 86.67057 & 86.66276 & 86.72005 & 86.75781 & 86.71875 & 86.6875 & 86.58984 & 86.66016 & 86.63281 \\
\hline & 3 & 81.03906 & 81.07161 & 81.17057 & 81.10026 & 81.14193 & 81.16927 & 81.11458 & 81.1224 & 81.11589 & 81.17318 \\
\hline & 4 & 80.38411 & 80.31771 & 80.35026 & 80.47266 & 80.46484 & 80.4401 & 80.41536 & 80.46615 & 80.44531 & 80.27604 \\
\hline & 5 & 44.80078 & 44.75781 & 44.73568 & 44.76432 & 44.71224 & 44.70573 & 44.80339 & 44.78125 & 44.75911 & 44.7474 \\
\hline & 6 & 79.38932 & 79.3151 & 79.28906 & 79.39844 & 79.45833 & 79.46484 & 79.40104 & 79.28516 & 79.28646 & 79.43099 \\
\hline & 7 & 64.83724 & 64.82422 & 64.84766 & 64.91276 & 64.86979 & 64.86979 & 64.86719 & 64.83854 & 64.86589 & 64.82031 \\
\hline & 8 & 57.54427 & 57.5013 & 57.48438 & 57.50391 & 57.55339 & 57.55859 & 57.56641 & 57.53125 & 57.5625 & 57.58464 \\
\hline \multirow{9}{*}{$\begin{array}{l}\text { Bit rate } \\
\text { (Mbps) }\end{array}$} & 0 & 2.01664 & 2.02961 & 2.018738 & 2.01664 & 2.009964 & 2.019882 & 2.025604 & 2.033615 & 2.030182 & 2.031708 \\
\hline & 1 & 2.013206 & 2.017784 & 2.017593 & 2.01931 & 1.995087 & 2.005196 & 2.01416 & 2.01683 & 2.01664 & 2.015495 \\
\hline & 2 & 1.945496 & 1.952553 & 1.953697 & 1.945305 & 1.939774 & 1.945496 & 1.950073 & 1.964378 & 1.954079 & 1.958084 \\
\hline & 3 & 2.777481 & 2.772713 & 2.758217 & 2.768517 & 2.762413 & 2.758408 & 2.766418 & 2.765274 & 2.766228 & 2.757835 \\
\hline & 4 & 2.873421 & 2.883148 & 2.87838 & 2.860451 & 2.861595 & 2.865219 & 2.868843 & 2.861404 & 2.864456 & 2.889252 \\
\hline & 5 & 8.085823 & 8.092117 & 8.09536 & 8.091164 & 8.098793 & 8.099747 & 8.085442 & 8.088684 & 8.091927 & 8.093643 \\
\hline & 6 & 3.019142 & 3.030014 & 3.033829 & 3.017807 & 3.009033 & 3.00808 & 3.017426 & 3.034401 & 3.03421 & 3.013039 \\
\hline & 7 & 5.150795 & 5.152702 & 5.149269 & 5.139732 & 5.146027 & 5.146027 & 5.146408 & 5.150604 & 5.146599 & 5.153275 \\
\hline & 8 & 6.219101 & 6.225395 & 6.227875 & \begin{tabular}{|l|}
6.225014 \\
\end{tabular} & 6.217766 & 6.217003 & 6.215858 & 6.221008 & 6.216431 & 6.213188 \\
\hline
\end{tabular}




\section{Mode 0}

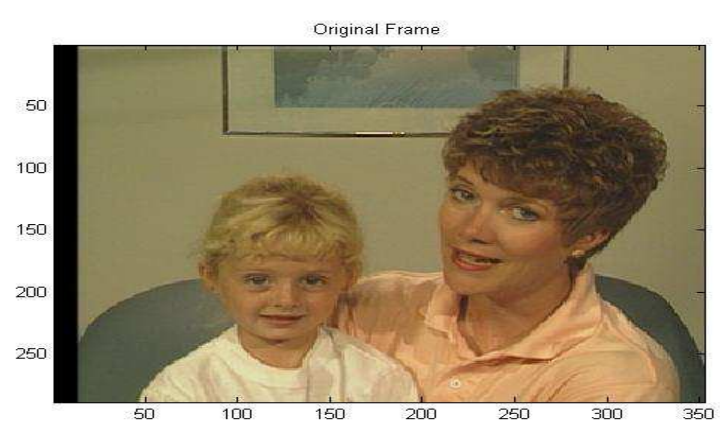

Fig.4. Original frame

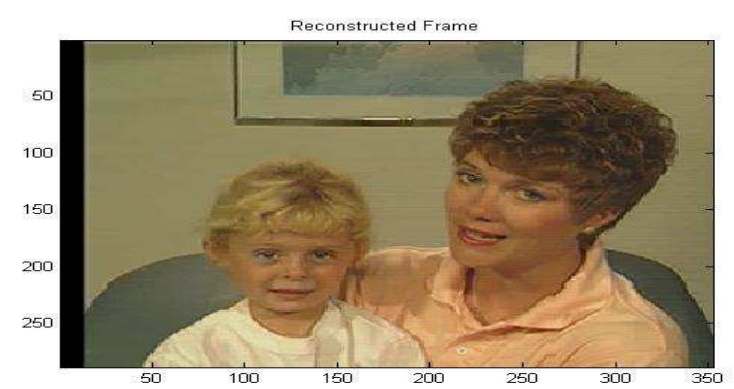

Fig.5. Reconstructed frame

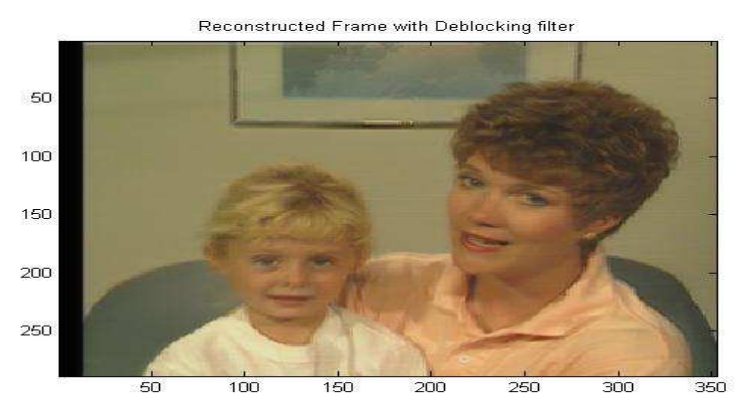

Fig.6. Reconstructed frame with Deblocking-filter

Mode 1

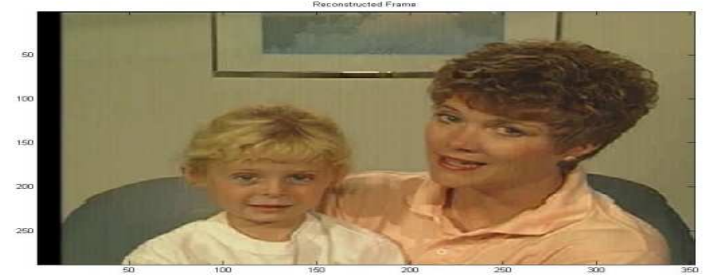

Fig.7. Reconstructed frame
Mode 2

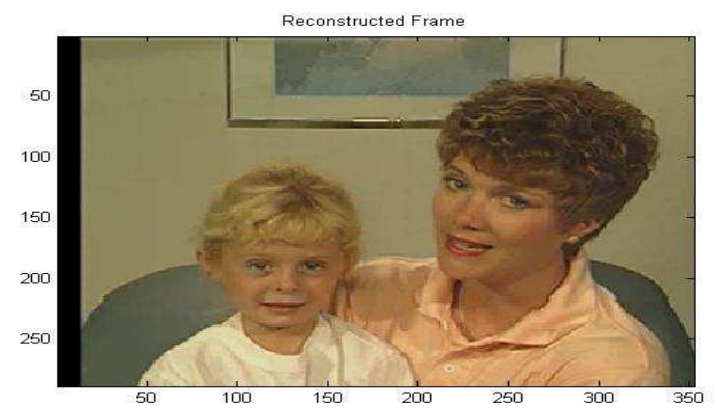

Fig.8. Reconstructed frame

Mode 3

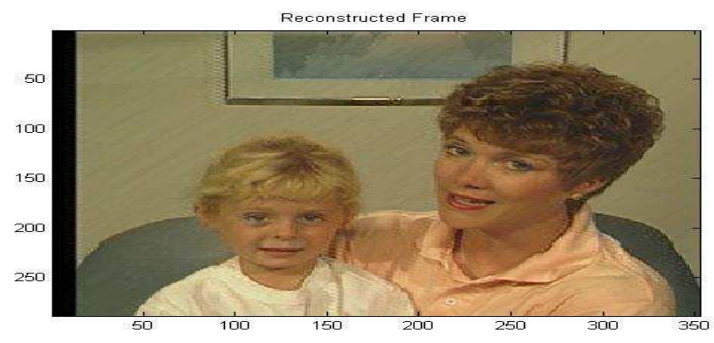

Fig.9. Reconstructed frame

Mode 4

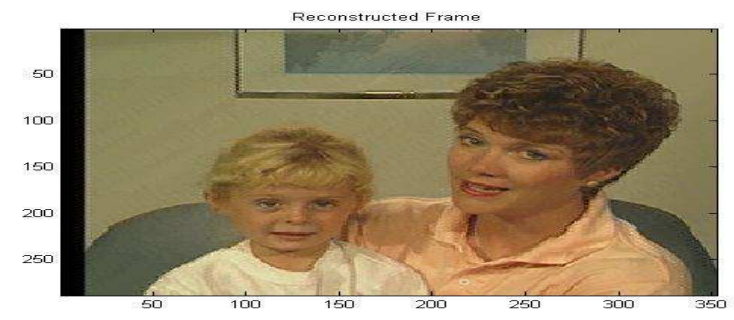

Fig 10. Reconstructed frame

Mode 5

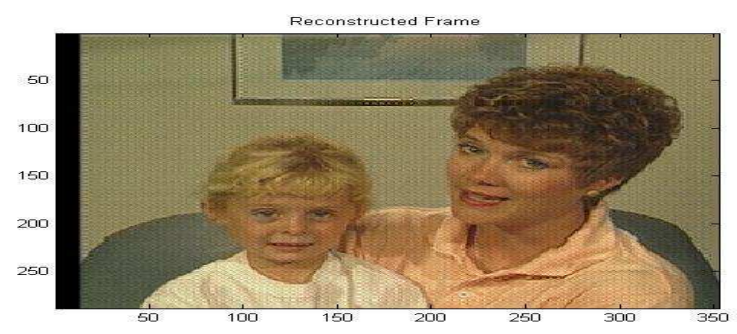

Fig.11. Reconstructed frame 


\section{Mode 6}

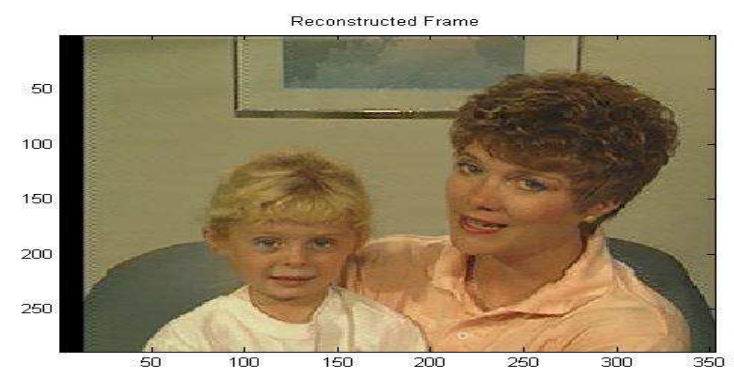

Fig.12 Reconstructed frame

\section{Mode 7}

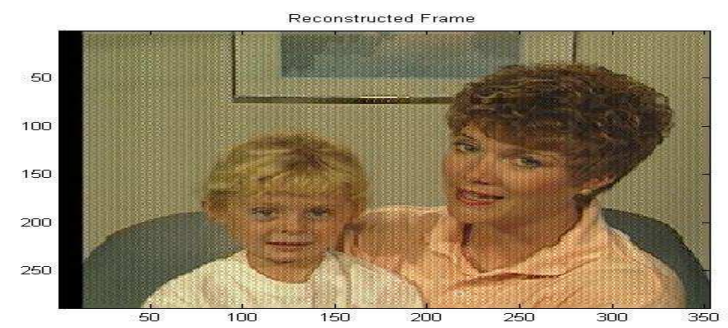

Fig.13. Reconstructed frame

\section{Mode 8}

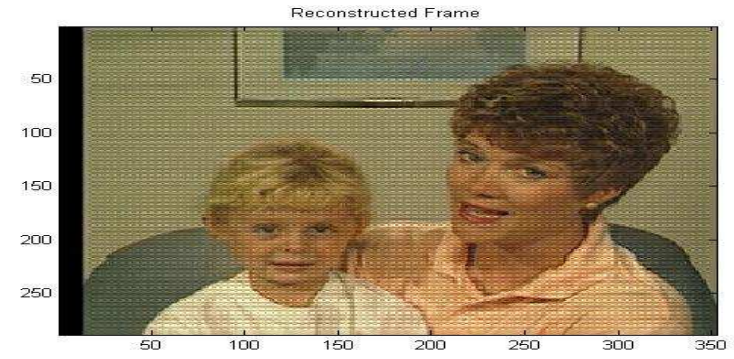

Fig.14. Reconstructed frame

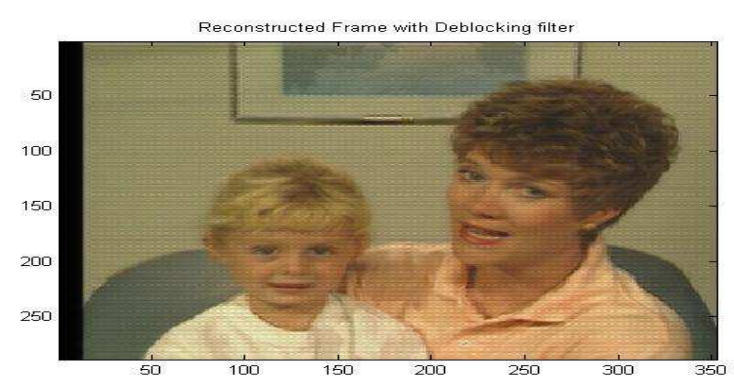

Fig.15. Reconstructed frame with Deblocking-filter

\section{Mode 0}

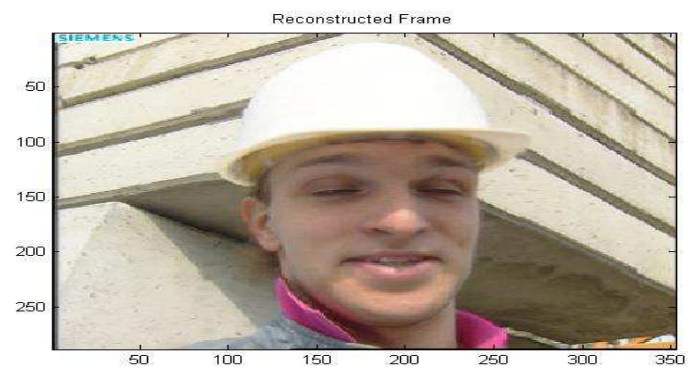

Fig.16. Reconstructed frame

Mode 1

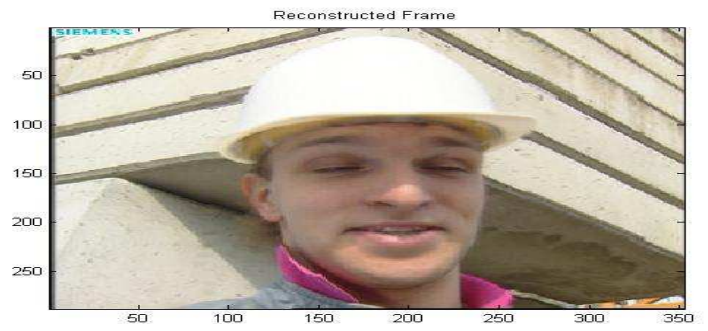

Fig.17. Reconstructed frame

Mode 2

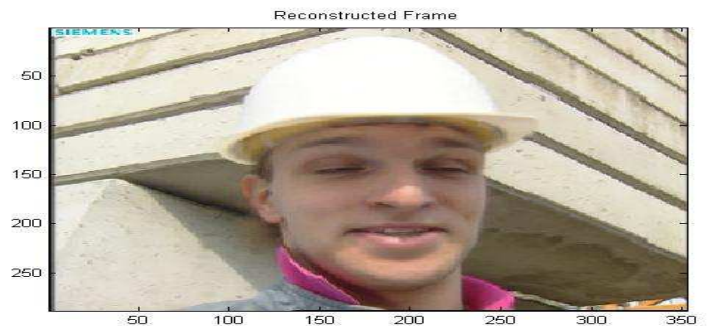

Fig.18. Reconstructed frame

Mode 7

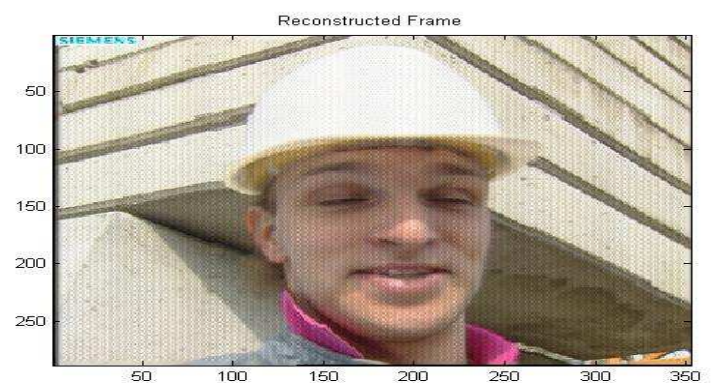

Fig.19. Reconstructed frame 


\section{Mode 8}

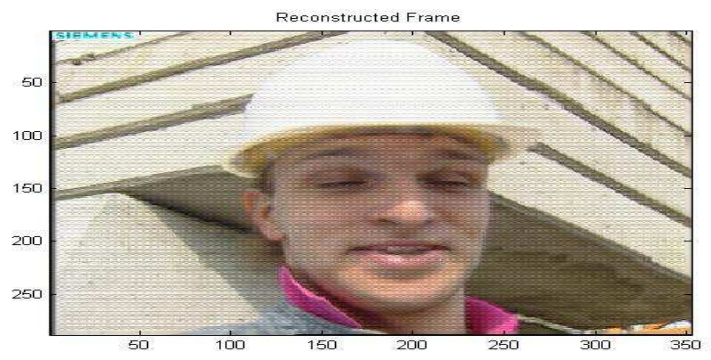

Fig.20. Reconstructed frame

\section{Mode 0}

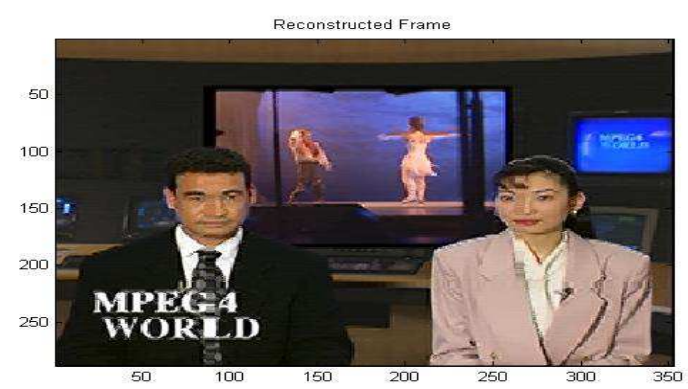

Fig.21. Reconstructed frame

\section{Mode1}

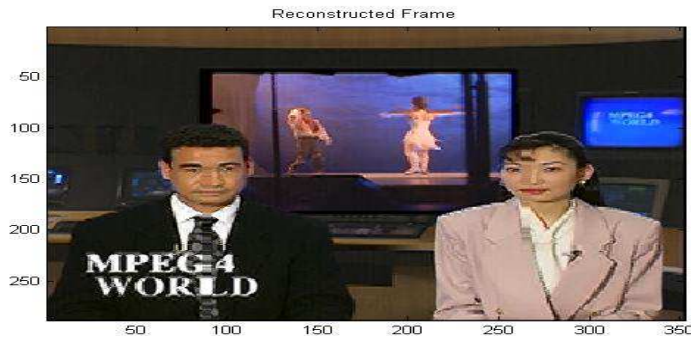

Fig.22. Reconstructed frame

\section{Mode2}

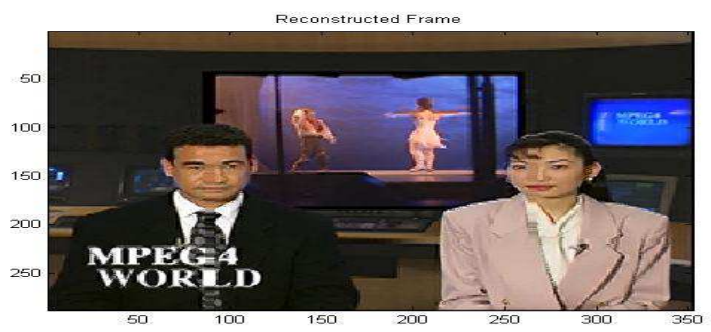

Fig.23. Reconstructed frame

\section{Mode 7}

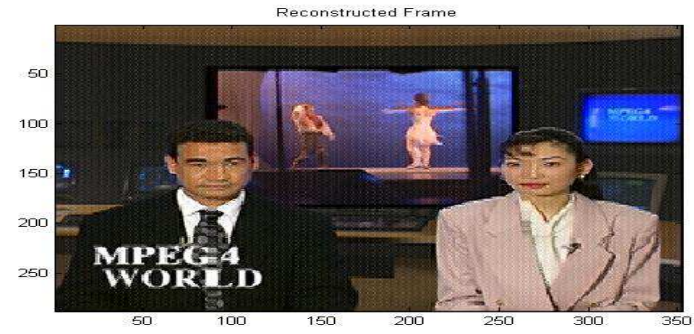

Fig.24. Reconstructed frame

\section{Mode 8}

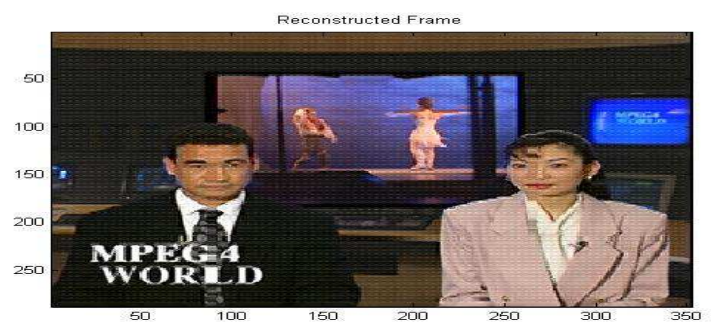

Fig.25. Reconstructed frame

\section{CONCLUSIONS}

The proposed work is selection of intra prediction modes and best prediction mode for intra frame coding in advanced video coding standard is carried out using Matlab. The results shows that the reconstructed picture quality (PSNR), compression ratio and bit rate achieved for test yuv I-frames, CIF format (mother-daughter and foreman and news) for different quantization parameters. Obtained results for all the nine intra prediction modes. The mode 0 , mode1, mode2, mode3, mode4 and mode6 gives good PSNR, compression ratio and low bit rate (tables I And II) compared to mode 5, mode7 and mode 8 . In the proposed method The mode 0 , mode1, mode2, mode 3 , mode4 and mode6 gives good PSNR, compression ratio and low bit rate, out of these modes mode 2 gives high PSNR, compression ratio and Low Bit rate indicted yellow mark in table(I and II), which is the best prediction mode.

\section{REFERENCES}

[1]. Iain E.Richardson,The H.264 and MPEG-4 Video Compression :Video coding for Next-generation Multimedia, Johan Wiley\& Sons, first edition 2003.

[2]. Iain E. Richarson, The H.264 Advanced Video Compression Standard, Johan Wiley\& Sons, Second edition 2010.

[3]. Youn-Long Steve Lin, Chao-Yang Kao Hung-Chih Kuo VLSI Design for Video Coding, Springer-2010. 
[4]. Huang Hui, Cao Tie-Yong, Zhang Xiong-wei, The Enhanced Intra Prediction Algorithm for H.264,congress on Image and signal processing 2008.

[5]. Chaminda Sampath Kannangara, Complexity Management of H.264/AVC Video Compression, the Robert Gordon University 2006.

[6]. http://www.vcodex.com 